\title{
ECT Image Recognition of Pipe Plugging Flow Patterns Based on Broad Learning System in Mining Filling
}

\author{
Xuebin Qin $\mathbb{D}^{1},{ }^{1}$ ChenChen Ji, ${ }^{1}$ Yutong Shen $\mathbb{D}^{1},{ }^{1}$ Pai Wang ${ }^{\circ},{ }^{1}$ Mingqiao Li $\mathbb{D},{ }^{1}$ \\ and Junle Zhang ${ }^{2}$ \\ ${ }^{1}$ College of Electrical and Control Engineering, Xi'an University of Science and Technology, 58 Yanta Road, Xi'an 710054, China \\ ${ }^{2}$ Shaanxi Institute of Metrology Science, Dongyi Road, Yanta District, Xi'an 710065, China \\ Correspondence should be addressed to Xuebin Qin; qinxb@xust.edu.cn
}

Received 31 December 2020; Revised 5 March 2021; Accepted 29 March 2021; Published 12 April 2021

Academic Editor: Lijie Guo

Copyright (C) 2021 Xuebin Qin et al. This is an open access article distributed under the Creative Commons Attribution License, which permits unrestricted use, distribution, and reproduction in any medium, provided the original work is properly cited.

\begin{abstract}
The process of mining filling, when the slurry is transported to the goaf by the filling pipeline, is very important to find the location and size of the caking in the filling pipeline in time for the safe and stable operation of the mine filling pipeline. It is an important research work to detect different flow patterns after two-dimensional section reconstruction in closed filling pipeline based on ECT (electrical capacitance tomography) visualization method. Slurry flow in pipeline is regarded as a two-phase flow, and the multishape distribution was reconstructed into images by ECT and intelligently recognized by broad learning system (BLS) algorithm. BLS is a feedforward neural network with few optimization parameters and fast training speed. In this paper, three features of two-phase sample images, the number of regional blocks, the roundness of regional blocks, and barycenter of regional blocks, are combined with network structure of BLS to recognize different flow patterns. Through the simulation, the recognition accuracy of two-phase fillback image is more than $99 \%$. This conclusion indicates the effectiveness of BLS to predict different twophase flow patterns; it also provides a new solution for the pattern recognition of the flow pattern in the mining filling pipeline.
\end{abstract}

\section{Introduction}

In the filling pipeline from the surface to the goaf, due to the unstable slurry flow pattern, there is a large density difference between the upper and lower parts of the pipe. The transport velocity is too low to make the coarse slurry deposit at the bottom of the pipe, which can easily cause serious blockage in the junction section between the horizontal pipeline and the vertical (or inclined) pipeline $[1,2]$. ECT visualization and BLS image recognition technology are helpful for rapid detection and slurry location in pipe blockage.

As slurry materials and proportions, strength and other factors lead to different flow conditions [3]; ECT visualization of pipeline cross section focuses on the permittivity of the medium in backfill pipeline. A possible option is to dynamically observe the internal conditions of the pipeline in the area where the blockage is most likely to occur a priori. ECT is a visualization technique for two-dimensional or three-dimensional image reconstruction of limited mediums or mixtures in a closed container [4]. ECT technology has the advantages of high accuracy and low cost. Its hardware mainly includes capacitive sensor, acquisition circuit, and computer. The capacitive sensor may be composed of 8 or 12 or 16 electrodes, in which each electrode travels around the closed container at an equal distance and is attached to the outer wall of backfill pipeline. When the ECT system is working, a set of independent capacitance data is collected through different excitation orders of electrodes to the computer for image reconstruction. From this set of independent capacitance data, the permittivity distribution of the slurry flow is inverted by image reconstruction algorithm. ECT technology has great potential for applications such as multiphase flow detection [5], combustion imaging [6], and fluidized bed solid particle monitoring [7]. In this paper, ECT technology is used to detect the insulation of mining fillings.

The classic image reconstruction algorithms of ECT technology include linear back projection (LBP) [8], Landweber [9], and Tikhonov regularization [10]. These 
algorithms have advantages and disadvantages. For example, using LBP algorithm has fast imaging speed but low accuracy, while the result of using the Landweber algorithm is that the image quality depends on parameters. Accuracy and efficiency have become the main obstacle for the field application of ECT technology, and it is also the main research direction of relevant scientific researchers. Wang Pai used least squares-support vector machines (LS-SVM) method to predict the capacitance error caused by the soft field, the fitness function of annealing particle swarm optimization (APSO) algorithm is constructed according to the capacitance error, and the image reconstruction with high precision is realized by searching for the best resolution [11]. Zhang proposed the ECT image reconstruction algorithm based on the compressed sensing theory and used the discrete Fourier transform basis to sparse the original image gray signal, and $l_{1}$ - norm regularization and primal-dual interior point method were used to reconstruct the image [12]. ECT is becoming more mature with the advent of many fast and accurate reconstruction algorithms.

ECT algorithms include forward and inverse problem solving. The forward problem refers to the establishment of mathematical model based on the distribution of permittivity in sensitive field and boundary conditions of sensitive field so as to obtain the mutual capacitance between electrode pairs. The inverse problem is to reconstruct the distribution of permittivity in sensitive field by the sensitivity of the field and the measured capacitance data and take the image as the final representation. However, the unprocessed reconstructed images cannot meet the requirements of the site. In order to achieve intelligent and integrated operation flow, ECT technology still needs various postprocessing, among which flow pattern recognition is the basic requirement. AbdeelJ Roman extracted the features of the space average permittivity and the position of the center of mass and combined them and used neural network to automatically identify the liquid-vapor two-phase flow patterns of refrigerant in horizontal tubes. The final experiment showed that the classification results of the nine flow patterns reached an average accuracy of $98.1 \%$ [13]. By using long short-term memory (LSTM) method, which can overcome the gradient disappearance or escalation, Rafael Johansen provided a successful application scenario for the flow pattern identification of annular, plug, slug, stratified, and wavy flow regimes [14]. Image reconstruction of twophase flow and flow pattern identification as a link in the field assembly line, their training speed, and classification accuracy has important influence on the subsequent operation.

In this paper, the ECT system is used to reconstruct the two-dimensional cross-sectional image inside the mining filling pipeline, and the broad learning system (BLS) provides a new idea for the flow pattern recognition inside the filling pipeline [15].

BLS is a novel neural network framework, which has the advantages of fewer parameters to be optimized and shorter training time compared with deep structural neural network. The simple BLS network is suitable for processing small sample sets of low dimensions and establishing online learning model efficiently. BLS network structure is flexible, and it can be used in combination with other networks. For example, the features extracted by convolutional neural network $(\mathrm{CNN})$ are combined with the output of BLS feature layer to enhance the ability of network prediction or classification under the premise of optimal training time. In this paper, the idea of combining features is adopted to realize flow pattern identification.

This paper is organized as follows: in Section 2, the basic principles of BLS theory are introduced. In Section 3, three artificial feature extraction methods are described. In Section 4, artificial feature extraction method and BLS are combined to enhance the ability of model recognition. The reconstruction results are analyzed and evaluated through simulation in Section 5. Concluding remarks are presented in Section 6.

\section{BLS Theory}

The predecessor of BLS is random vector functional-link neural network [16] (RVFLNN), which essentially belongs to feedforward neural network (FNN). BLS network consists of mapping layer, enhancement layer, and output layer. A BLS network is shown in Figure 1. First, input data is mapped to "feature nodes" through sparse weight mapping, and these "feature nodes" form the mapping layer. Second, the output of the mapping layer serves as the input of the enhancement layer and is activated by the "enhancement node." Finally, the output of the mapping layer and the output of the enhancement layer are combined as the input vector of the output network, and the corresponding weight is calculated by the Moore-Penrose generalized inverse.

The main network relations of each layer are as follows:

$$
\begin{aligned}
\text { mapping layer : } Z & =\phi\left(X W_{e}+\beta_{e}\right), \\
\text { enhancement layer : } H & =\xi\left(Z W_{h}+\beta_{h}\right), \\
\text { output layer }: Y & =[Z \mid H] W^{m},
\end{aligned}
$$

where $X$ is the input data; $Y$ is known label data; $\phi(\cdot)$ is the identity mapping function; $\xi(\cdot)$ is the tan sig activation function $\left(\tan \operatorname{sig}(x)=\left(2 /\left(1+e^{-2 x}\right)\right)-1\right) ; W_{e}, W_{h}$, and $W^{m}$ are weights of mapping layer, enhancement layer, and output layer, respectively; $\beta_{e}$ and $\beta_{h}$ are biases; $Z$ and $H$ are the outputs of the mapping layer and the enhancement layer, respectively.

Set the input data to $X_{m \times n}$ ( $m$ is the number of samples in the training set and $n$ is the number of pixels). A column vector whose entries are all constants adds to the end of $X_{m \times n}$ and the new matrix is written as $X_{m \times(n+1)}$, so equation (1) can be abbreviated as

$$
Z=\phi\left(X W_{e e}\right)
$$

where $W_{e e}$ is a randomly generated $(n+1) \times N_{1}$ weight matrix $\left(N_{1}\right.$ is the number of "feature nodes" in the mapping layer). It is a necessary processing to sparse $W_{e e}$ to effectively reduce the linear correlation of new "feature nodes."

The output $Z_{m \times N_{1}}$ of the mapping layer is the input to the enhancement layer. Similarly, equation (1) can be abbreviated as 


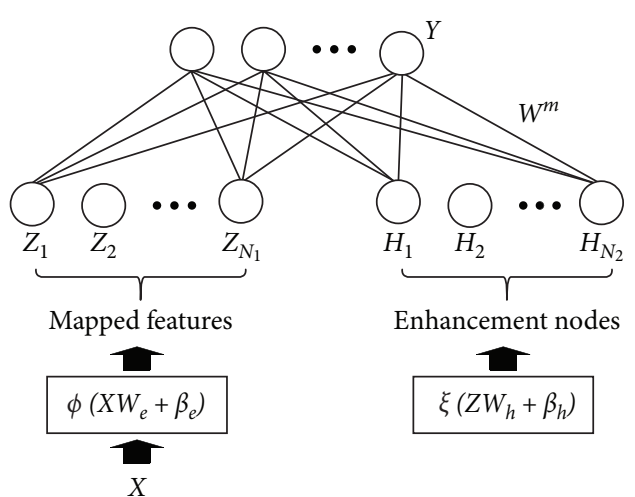

Figure 1: Network model of BLS.

$$
H=\xi\left(Z W_{h h}\right)
$$

where $W_{h h}$ is the randomly generated weight matrix of $\left(N_{1}+1\right) \times N_{2}\left(N_{2}\right.$ is the number of "enhancement nodes" of the enhancement layer). The orthonormal basis of $W_{h h}$ was used to improve the feature extraction ability of the enhancement layer. $[Z \mid H]_{m \times\left(N_{1}+N_{2}\right)}$ is obtained by combining the output features of the mapping layer and the enhancement layer. $A\left(A=[Z \mid H]_{m \times\left(N_{1}+N_{2}\right)}\right)$ directly establishes the mapping equation (1) with label data $Y$. In order to solve the weight $W^{m}$ of the output layer, the optimization of equation (1) is established as

$$
\underset{W^{m}}{\arg \min }\left\|Y-A W^{m}\right\|_{2}^{2}+\lambda\left\|W^{m}\right\|_{2}^{2},
$$

where $\lambda$ is a parameter close to the $l_{2}$ - norm and regularization method is adopted to prevent model overfitting.

To solve for weight $W^{m}$ is to solve for the Moore-Penrose generalized inverse.

$$
W^{m}=\left(\mathrm{A}^{T} \mathrm{~A}+\lambda \mathrm{I}\right)^{-1} A^{T} Y
$$

The BLS network model is mainly composed of $W 1$ of the mapping layer, $W 2$ of the enhancement layer, and $W 3$ of the output layer. Since there is no need for backpropagation during training, the training time is very short. In addition, BLS can provide incremental learning similar to migration learning when dealing with big data, including incremental enhancement node, incremental feature node, and incremental input data. Incremental learning avoids data retraining and greatly saves training time.

\section{Feature Extraction}

In this paper, the BLS network is used to identify the flow pattern inside the mining filling pipeline, and the characteristics of the filling body are added to the branches of the original BLS network to further improve the recognition accuracy. These are three different functions and their extraction methods.

3.1. Number of Regional Blocks. Obtaining the number of area blocks is an important index to judge the pipeline blockage. Based on OpenCV library, the binary image of two-phase flow is used to obtain the number of blocks in the pipeline.

3.2. Circularity of Regional Block. Block shape complexity can be described by shape coefficient. For example, the circularity coefficient of stratified flow is small, while that of bubble flow is large.

$$
f f=4 \pi \cdot \frac{S}{C^{2}}
$$

where $S$ represents the area of the block and $C$ represents the perimeter of the block.

3.3. Barycenter of Regional Block. Barycenter reflects the location information of the region block. For example, pixels barycenter of stratified flow is near the bottom of the pipe, and pixels barycenter of circulation is generally near the center. Barycenter position of the block is calculated by means of moment based binary image of two-phase flow section [17].

$$
\begin{aligned}
M_{00} & =\sum_{I} \sum_{J} v(i, j), \\
M_{10} & =\sum_{I} \sum_{J} i \cdot v(i, j), \\
M_{01} & =\sum_{I} \sum_{J} j \cdot v(i, j), \\
x_{c} & =\frac{M_{10}}{M_{00}}, \\
y_{c} & =\frac{M_{01}}{M_{00}},
\end{aligned}
$$

where $M_{00}$ is the area of the white area of the image block. $v(i, j)$ represents the pixel value of the white region on the binary image, where $(i, j)$ is the coordinate on the image; $M_{10}$ and $M_{01}$, respectively, represent the accumulation of coordinate values in the $x$ direction and $y$ direction of the white area on the binary image. $\left(x_{c}, y_{c}\right)$ are the barycentric coordinates.

\section{Proposed Method}

For the three features extracted from sample sets, the number of regional blocks, circularity, and the barycentric coordinates are represented by $P_{1}, P_{2}, P_{3}$, respectively. After combining them, $P=\left[\begin{array}{lll}P_{1} & P_{2} & P_{3}\end{array}\right]$ is obtained, where $P_{1}, P_{2}, P_{3}$ is the matrix of $m \times 1, m \times q$, and $m \times 2 q$, respectively, $m$ is the number of sample sets, and the value of $q$ is equal to the number of categories to be classified. The number of categories in this article is 6 ; then $q=6$ is set.

If $P^{(i)}=\left[\begin{array}{ccc}P_{1}^{(i)} & P_{2}^{(i)} & P_{3}^{(i)}\end{array}\right]$ calculated from the ith sample image, then the calculated value of the circular degree corresponding to the sample is placed in the front position of matrix $P_{2}^{(i)}$, and the remaining position is filled with 0 ; the 
barycentric coordinate is placed in the front position of matrix $P_{3}^{(i)}$, and the remaining position is filled with 0 .

As shown in Figure 2, the BLS mapping layer is augmented with "feature node," and the output of the mapping layer is combined with the feature matrix $P$ to form a new matrix $[Z \mid P]$. After the normalization of $P$, the input of BLS's enhancement layer is also $[Z \mid P]$ :

$$
\begin{aligned}
H^{*} & =\xi\left([Z \mid P] W_{h h}\right), \\
Y & =\left[Z|P| H^{*}\right] W^{m}=A^{*} W^{m},
\end{aligned}
$$

where $H^{*}$ is the output of the enhancement layer; $A^{*}=\left[Z|P| H^{*}\right]$, representing the input to the output layer.

Additional features are added to the BLS architecture to ensure the accuracy of identification; and because the amount of data for additional features is small, it hardly affects the training speed.

\section{Simulation and Experiment}

5.1. The Data Set. The data set in this article simulates ECT to reconstruct a two-dimensional cross-sectional image of a mining filling pipeline, and the size of each sample image is $64 \times 64$ pixels. There are 6 types of flow patterns: single bubble, double bubbles, three bubbles, four bubbles, stratified, and annular flow. The data set includes training set, training set label, test set, and test set label. The training set has a total of 3000 images, including 500 images of different categories. The test set has a total of 500 images, with 100 images for each category. Part of the training set is shown in Figure 3.

After the pixels of the sample image are compressed to $32 \times 32$ pixels, and row by row into a 1 by 2 row vector $1 \times 1024$, the training set is a $3000 \times 1024$ matrix and the test set is a $500 \times 1024$ matrix.

The training set label and the test set label are matrices of $3000 \times 6$ and $500 \times 6$, respectively. The elements of the label matrix are 0 and 1 . The row corresponds to different samples and the column corresponds to different categories.

5.2. Feature Representation. Based on the feature extraction method proposed in Section 4, three features are extracted from the reconstructed binary image by ECT. Figure 4 shows a sample of stratified flow. The image size is $64 \times 64$ pixels. The number of regional blocks is 1 , the barycenter coordinate is $(31,45)$, and the circularity is 0.5344 . It can be seen from this sample that the barycenter of the stratified flow image is downward, and the circularity is small.

5.3. Simulation Result. In order to verify the influence of additional features on BLS network flow pattern identification, this paper discusses three groups of schemes, namely, BLS network to train data, the combination of BLS networks with individual features to train data, and the combination of BLS networks with three features to train data.

5.3.1. BLS Network Training Results. Adjust the number of feature nodes and enhancement nodes in BLS network, and the training accuracy, test accuracy, and training time are shown in Table 1.

As shown in the first row of Table 1, the numbers of feature nodes and enhancement nodes were set as 200 and 2000, respectively, and the training accuracy was $99.57 \%$ after the training set data was input into the BLS network, and the training time was $0.5787 \mathrm{~s}$; the test accuracy is $66.96 \%$ after the test set data was input into BLS network.

In Table 1, the number of feature nodes was set to 200 . With the increase of the number of enhanced nodes, the test accuracy is improved by a small margin. The number of enhancement nodes was fixed at 10000 . With the increase of the number of feature nodes, the accuracy of the test set decreases by a small margin. This indicates that the enhancement layer of BLS greatly influences the training results. Since the activation function of BLS enhancement layer is $\tan \operatorname{sig}(\cdot)$, it has the ability to fit nonlinear functions, which is also the core function of BLS network.

The training accuracy of BLS network is close to $100 \%$, but the test accuracy is low, which is a typical overfitting phenomenon. There are two possible reasons for the overfitting phenomenon in BLS network:

(1) The mapping layer is a direct linear transformation system, whose extracted features are not sufficiently expressive or have few extracted features

(2) There are too many parameters in the enhancement layer, which overfits the training set, resulting in poor performance of the model on the test set

5.3.2. Combination of Features. The main reasons for the poor classification results of two-phase flow by single BLS network are the limitations of network architecture and insufficient feature extraction ability. In this section, the additional extracted features, including $P_{1}$ (the number of regional blocks), $P_{2}$ (the circularity of the regional blocks), and $P_{3}$ (the barycenter coordinates of the regional blocks), are combined with the "feature nodes" of the BLS mapping layer, respectively. The classification results of the two-phase flow by this network are shown in Table 2.

As shown in the first row of Table 2, 200 feature nodes and 2000 enhancement nodes were set, respectively. When feature nodes of BLS and $P_{1}$ are combined, the accuracy rate of test set is $76.73 \%$ and the training time is $0.6187 \mathrm{~s}$. When $P_{2}$ is combined with feature nodes, the accuracy of test set is $97.13 \%$ and the training time is $0.6754 \mathrm{~s}$. When $P_{3}$ is combined with feature nodes, the accuracy of test set is $97.13 \%$ and the training time is $0.6754 \mathrm{~s}$.

Combine the three additional features $P_{1}, P_{2}$, and $P_{3}$ into $P=\left[\begin{array}{lll}P_{1} & P_{2} & P_{3}\end{array}\right]$; three additional features, $P_{1}, P_{2}$, and $P_{3}$, are combined into $P=\left[\begin{array}{lll}P_{1} & P_{2} & P_{3}\end{array}\right]$, and $P$ is combined with feature nodes of BLS mapping layer. The identification results of the two-phase flow data set are shown in Table 3.

As shown in the first row of Table 3, the numbers of feature nodes and enhancement nodes were set as 200 and 2000 , respectively. The training accuracy was $99.68 \%$ and the training time was $0.6083 \mathrm{~s}$ after the training data was input 


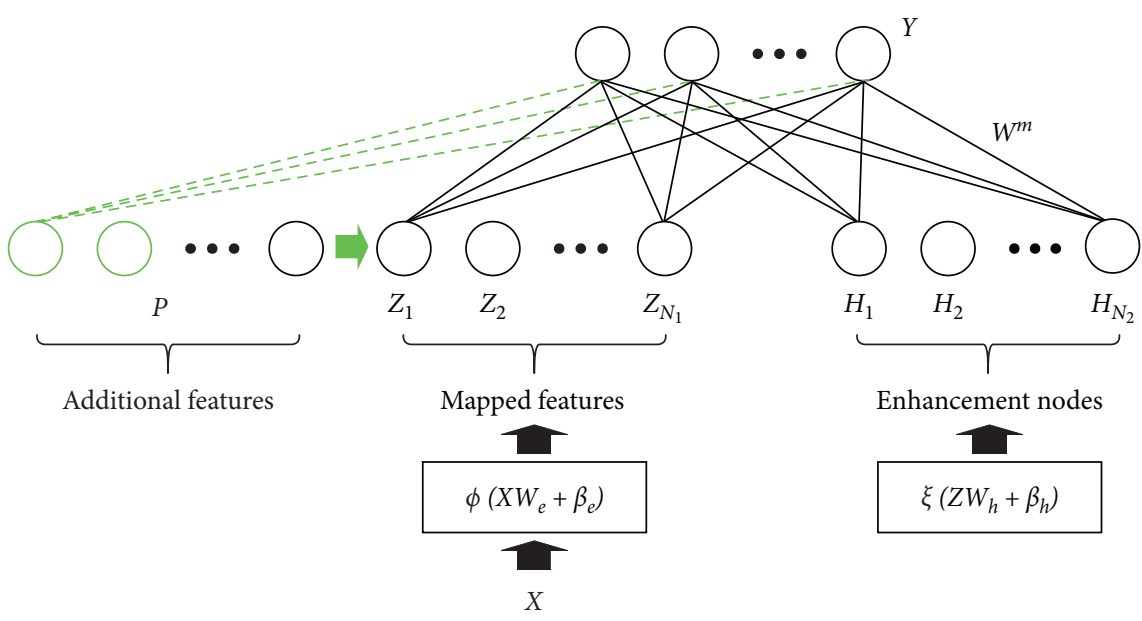

FIgURE 2: Network model of BLS.
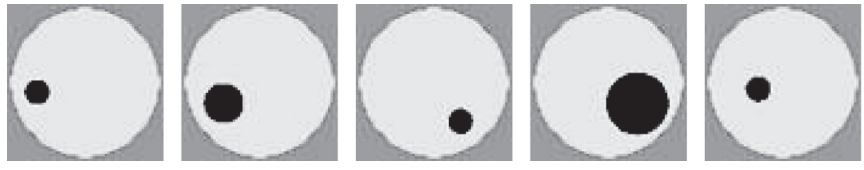

(a)
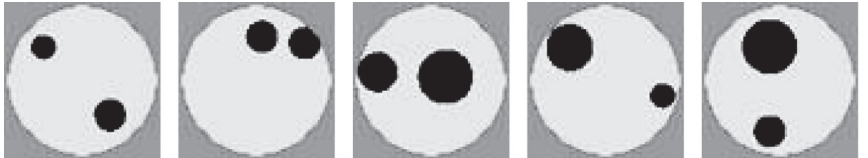

(b)
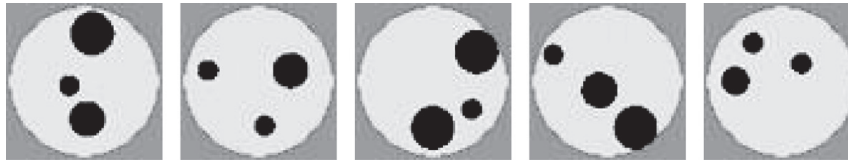

(c)
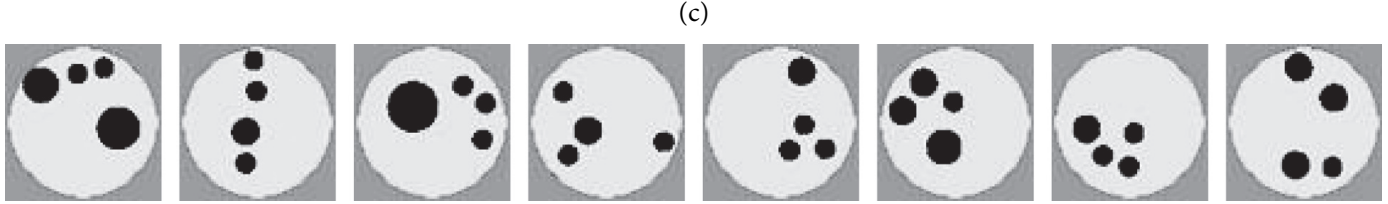

(d)
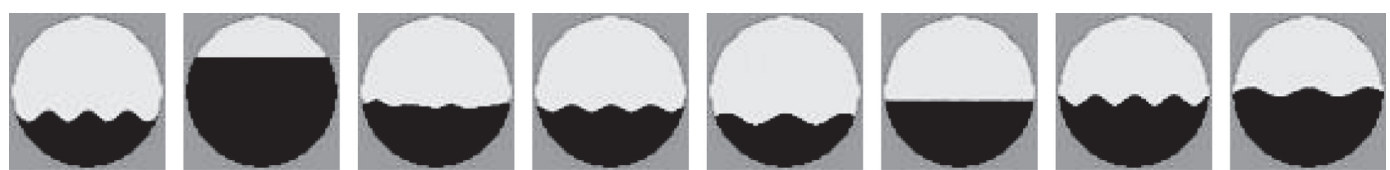

(e)
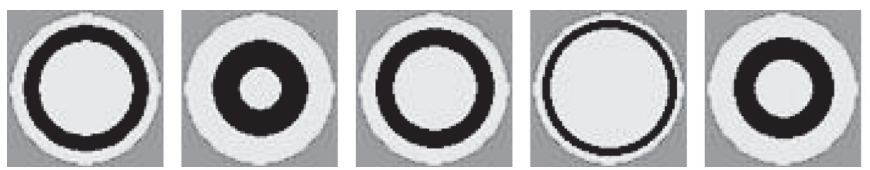

(f)
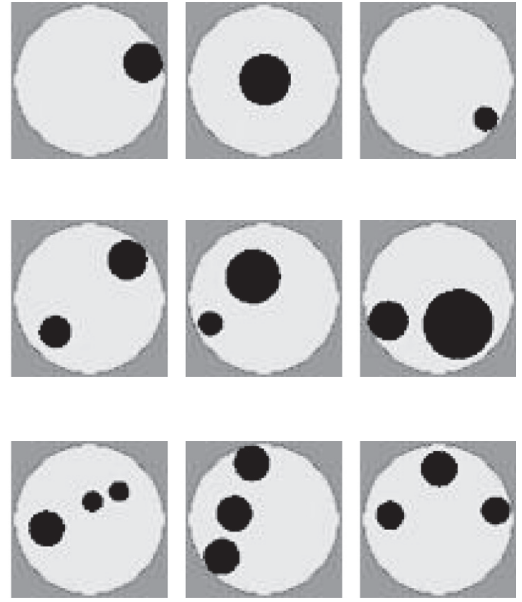

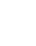




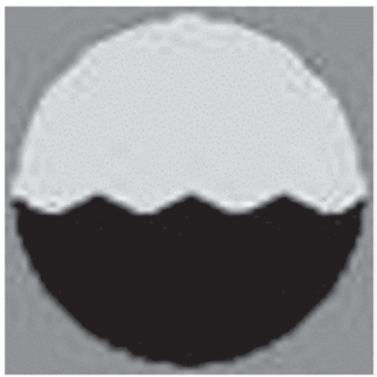

(a)

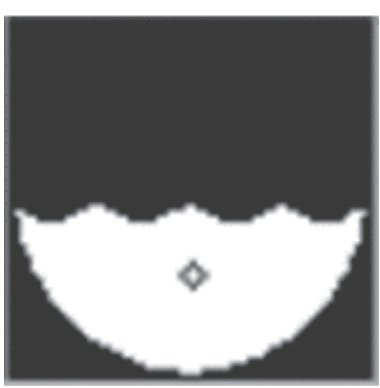

(b)

FIgURE 4: Feature extraction of ECT reconstructed images. (a) Reconstructed gray-scale image. (b) Reconstructed binary image.

TABLE 1: The result of BLS.

\begin{tabular}{lcccc}
\hline Feature nodes & Enhancement nodes & Training accuracy (\%) & Testing accuracy (\%) & Training time (s) \\
\hline 200 & 2000 & 99.57 & 66.96 & 0.5787 \\
200 & 4000 & 99.84 & 68.92 & 1.3383 \\
200 & 6000 & 99.87 & 69.98 & 2.1988 \\
200 & 8000 & 99.76 & 69.80 & 4.3135 \\
200 & 10000 & 99.91 & 70.34 & 6.6591 \\
200 & 12000 & 99.66 & 68.56 & 7.3596 \\
500 & 10000 & 99.53 & 70.34 & 7.0168 \\
2000 & 10000 & 99.85 & 67.14 & 14.5632 \\
4000 & 10000 & 99.72 & 68.21 & 45.6702 \\
\hline
\end{tabular}

TABLE 2: The result of adding one feature to BLS.

\begin{tabular}{|c|c|c|c|c|c|c|c|}
\hline \multirow{2}{*}{ Feature nodes } & \multirow{2}{*}{ Enhancement nodes } & \multicolumn{3}{|c|}{ Testing accuracy (\%) } & \multicolumn{3}{|c|}{ Training time (s) } \\
\hline & & $P_{1}$ & $P_{2}$ & $P_{3}$ & $P_{1}$ & $P_{2}$ & $P_{3}$ \\
\hline 200 & 2000 & 76.73 & 97.13 & 98.35 & 0.6187 & 0.6754 & 0.7246 \\
\hline 200 & 4000 & 82.06 & 97.28 & 98.52 & 1.1592 & 1.2627 & 1.3174 \\
\hline 200 & 6000 & 83.30 & 97.46 & 98.76 & 2.1641 & 2.3541 & 4.2512 \\
\hline 200 & 8000 & 83.84 & 97.59 & 98.89 & 4.3135 & 4.2429 & 4.5854 \\
\hline 200 & 10000 & 84.19 & 97.63 & 98.86 & 6.2257 & 6.2466 & 6.3350 \\
\hline 200 & 12000 & 82.95 & 97.35 & 98.49 & 9.3698 & 9.3321 & 9.1278 \\
\hline 500 & 10000 & 80.82 & 97.41 & 98.21 & 7.1576 & 7.2756 & 7.1853 \\
\hline 2000 & 10000 & 75.66 & 97.25 & 98.43 & 14.2775 & 14.5853 & 14.4428 \\
\hline 4000 & 10000 & 73.89 & 97.33 & 98.37 & 46.4412 & 47.1185 & 47.6614 \\
\hline
\end{tabular}

TABLE 3: The result of adding three features to BLS.

\begin{tabular}{lcccc}
\hline Feature nodes & Enhancement nodes & Training accuracy (\%) & Testing accuracy (\%) & Training time (s) \\
\hline 200 & 2000 & 99.98 & 99.54 & 0.6083 \\
200 & 4000 & 99.94 & 99.67 & 1.2704 \\
200 & 6000 & 99.98 & 99.76 & 2.2515 \\
200 & 8000 & 99.95 & 99.79 & 4.0251 \\
200 & 10000 & 99.90 & 99.82 & 6.0906 \\
200 & 12000 & 99.96 & 99.65 & 9.1225 \\
500 & 10000 & 99.99 & 99.81 & 7.3056 \\
2000 & 10000 & 99.92 & 99.85 & 14.0437 \\
4000 & 10000 & 99.93 & 99.87 & 47.9779 \\
\hline
\end{tabular}

into BLS network; the test accuracy is $66.54 \%$ when test data was input into BLS network.

The results of Table 2 show that $P_{1}$ improves BLS network to some extent, but the effect is not significant; $P_{2}$ and
$P_{3}$ have significant improvement effect on BLS network and high accuracy of test set. The results of Table 3 show that the test accuracy is close to $100 \%$ when $P_{1}, P_{2}$, and $P_{3}$ jointly affect the BLS network. The training time of the BLS network 
with the additional features is similar to that of the single BLS network, which hardly affected the training speed.

\section{Summary and Prospect}

In order to better analyze the distribution of blockage material in filling pipeline comprehensively, this paper proposes to adopt ECT method to observe the blockage area visually. According to the permittivity of the plugging material, six shapes of fluids (single-bubble, double-bubble, three-bubble, four-bubble, stratified, and annular flow) in the cross-sectional view are simulated. A fusion method of artificial features and BLS was proposed to recognize the flow pattern of blocked pipes in the gray image reconstructed by ECT. The training set and test set have 3000 and 500 sample images, respectively. In the simulation, the BLS network that can be quickly trained is used to identify the flow pattern of two-phase flow images. Due to the lack of feature extraction ability of BLS network, the simulation results show that the identification effect of test set is poor. In view of this situation, this paper used the method of nonneural network to extract three additional features, which are the number of regional blocks, the circularity of regional blocks, and the barycenter coordinates of regional blocks. Three different features are combined with feature nodes of BLS mapping layer to realize high accuracy test set recognition. In addition, the training time is almost unaffected because of the small amount of data of the additional features selected. Further work is to extract fewer and more prominent features from ECT reconstructed images, thereby improving the accuracy and speed of recognition.

\section{Data Availability}

The data used in this paper were randomly generated by programming and simulation software, including the training set and the test set (the reconstructed images of the ECT system were taken as samples).

\section{Conflicts of Interest}

The authors declare that they have no conflicts of interest.

\section{Acknowledgments}

This research was supported by the National Natural Science Foundation of China (no. 51704229) and the Natural Science Basic Research Plan of Shaanxi Province of China (no. 2019JM-074).

\section{References}

[1] R. Gao, K. Zhou, Y. Zhou, and C. Yang, "Research on the fluid characteristics of cemented backfill pipeline transportation of mineral processing tailings," Alexandria Engineering Journal, vol. 59, no. 6, pp. 4409-4426, 2020.

[2] J. Zhang, W. Guo, and H. Li, "Effects of backfill soil on pipeline's mechanical response subjected to perilous rock impact," Engineering Review, vol. 40, no. 1, pp. 25-31, 2020.

[3] C. Qi, A. Fourie, Q. Chen, and Q. Zhang, "A strength prediction model using artificial intelligence for recycling waste tailings as cemented paste backfill," Journal of Cleaner Production, vol. 183, pp. 566-578, 2018.

[4] M. Meribout and I. M. Saied, "Real-time two-dimensional imaging of solid contaminants in gas pipelines using an electrical capacitance tomography system," IEEE Transactions on Industrial Electronics, vol. 64, no. 5, pp. 3989-3996, 2017.

[5] Y. Shi, M. Wang, and M. Shen, "Characterization of oil-water two-phase flow in a horizontal pipe with multi-electrode conductance sensor," Journal of Petroleum Science and Engineering, vol. 146, pp. 584-590, 2016.

[6] Y. Jia and Q. Chen, "Theoretical analysis of the dielectric characteristics of plasma flame and imaging plasma flame using Electrical Capacitance Tomography," in Proceedings of the IEEE International Conference on Imaging Systems \& Techniques, Beijing, China, October 2013.

[7] X. Li, A. J. Jaworski, and X. Mao, "Bubble size and bubble rise velocity estimation by means of electrical capacitance tomography within gas-solids fluidized beds," Measurement, vol. 117, pp. 226-240, 2018.

[8] B. Matusiak, M. J. Da Silva, U. Hampel, and A. Romanowski, "Measurement of dynamic liquid distributions in a fixed bed using electrical capacitance tomography and capacitance wire-mesh sensor," Industrial \& Engineering Chemistry Research, vol. 49, no. 5, pp. 2070-2077, 2010.

[9] H. Yan, Y. F. Wang, and Y. G. Zhou, "Three-dimensional electrical capacitance tomography reconstruction by the Landweber iterative algorithm with fuzzy thresholding," IET Science Measurement \& Technology, vol. 8, no. 6, pp. 487-496, 2014.

[10] L. Wang, X. Liu, D. Chen, H. Yang, and C. Wang, "ECT image reconstruction algorithm based on multiscale dual-channel convolutional neural network," Complexity, vol. 2020, Article ID 4918058, 2020.

[11] Tsinghua University, Sensor Research; Reports from Tsinghua University Provide New Insights into Sensor Research (A Deep Learning Compensated Back Projection for Image Reconstruction of Electrical Capacitance Tomography), Tsinghua University, Beijing, China, 2020.

[12] Z. Cao, L. Ji, and L. Xu, "Iterative reconstruction algorithm for electrical capacitance tomography based on Calderon's method," IEEE Sensors Journal, vol. 18, no. 20, pp. 8450-8462, 2018.

[13] A. J. Roman, P. J. Kreitzer, J. S. Ervin, M. S. Hanchak, and L. W. Byrd, "Flow pattern identification of horizontal twophase refrigerant flow using neural networks," International Communications in Heat and Mass Transfer, vol. 71, pp. 254-264, 2016.

[14] R. Johansen, T. G. Ostby, A. Dupré, and S. Mylvaganam, "Long short-term memort neural networks for flow regime identification using ECT," in Proceedings of the 9th World Congress on Industrial Process Tomography, Bath, UK, September 2018.

[15] C. L. P. Chen and Z. Liu, "Broad learning system: an effective and efficient incremental learning system without the need for deep architecture," IEEE Transactions on Neural Networks and Learning Systems, vol. 29, no. 1, pp. 10-24, 2017.

[16] S. Feng and C. L. P. Chen, "Fuzzy Broad learning system: a novel neuro-fuzzy model for regression and classification," IEEE Transactions on Cybernetics, vol. 50, no. 2, pp. 414-424.

[17] T. Tibyani and S. I. Kamata, "Registering 3D objects triangular meshes using an interest point detection on barycentric coordinates," in Proceedings of the 2012 International Conference on Informatics, Electronics \& Vision (ICIEV), Dhaka, Bangladesh, May 2012. 